Check for updates

Cite this: Mater. Adv., 2020, 1,1763

\section{Advanced development of a non-ionic surfactant and cholesterol material based niosomal gel formulation for the topical delivery of anti-acne drugs}

\author{
Akhilesh Shah, ${ }^{a}$ Sanjay Boldhane, ${ }^{b}$ Atmaram Pawar (D) ${ }^{a}$ and \\ Chellampillai Bothiraja (D) *a
}

\begin{abstract}
The aim of the present investigation was to develop adapalene (ADP), a high lipophilicity and low solubility anti-acne drug-loaded niosomal topical gel formulation, in order to improve its therapeutic efficacy. ADP niosomes were prepared using Span 60 and cholesterol using a modified ethanol injection method. A design of experiments (DOE) study was conducted to optimize ADP loaded niosomes. The potential of ADP niosomes was investigated for in vitro release and ex vivo skin permeation studies. Additionally, ADP niosomes were loaded into the Carbopol $934 \mathrm{gel}$ and studied for its skin irritation, skin deposition and ex vivo skin permeation potential. The developed ADP niosomes showed the mean particle size, zeta potential, and entrapment efficiency of $278 \mathrm{~nm},-17.99 \mathrm{mV}$, and $86 \%$, respectively. The optimized ADP niosomes showed controlled drug release up to $12 \mathrm{~h}$. Nevertheless, the niosomal gel displayed controlled drug release up to $24 \mathrm{~h}$ with a reduction in skin irritation in Wistar rats. An in vivo skin deposition study showed 2.5-fold higher ADP retention in the stratum corneum layer as compared to the commercial ADP formulation. The ADP niosomal gel would be a safe and valuable alternative to the conventional delivery systems for the treatment of acne.
\end{abstract}

Received 11th May 2020 Accepted 13th July 2020

DOI: $10.1039 / \mathrm{d} 0 \mathrm{ma} 00298 \mathrm{~d}$

rsc.li/materials-advances

\section{Introduction}

Acne vulgaris is the most common and chronic skin disease in teenagers and adults. It is associated with an elevated rate of sebum secretion which appear as mild-severe inflammatory lesions on the areas of the face, back and chest enclosing large numbers of sebaceous follicles. It is categorized as mild, moderate or severe based on the number and type of lesions. Treatment choices differ with the phase and intensity of the disease. Usually, topical treatment is favored for mild and moderate acne while systemic therapy is utilized to treat severe cases. Topical therapy mainly contains retinoids (vitamin A), antibiotics and combinations thereof., ${ }^{1,2}$

Adapalene (ADP) is chemically known as 6-[3-(1-adamantyl)4-methoxyphenyl]-2-naphthoic acid. ADP is a synthetic third generation retinoid compound and a broad-spectrum anti-acne agent approved by the USFDA in 1996. Although the precise mechanism of action is unknown, it binds to certain retinoic acid receptors in the nucleus leading to specific gene expression.

\footnotetext{
${ }^{a}$ Department of Pharmaceutics, Poona College of Pharmacy, Bharati Vidyapeeth (Deemed to be University), Erandawane, Pune 411038, Maharashtra, India. E-mail: pounbothi@yahoo.com; Fax: + 9120 25439383; Tel: + 912025437237

${ }^{b}$ Formulation Development, Micro Labs Limited, Bangalore-560 001, India
}

It accelerates skin growth through the modulation of cellular differentiation, keratinization of follicular epithelial cells and inflammatory processes. ${ }^{3,4} \mathrm{ADP}$ is a BCS class II drug with $\log P$ and $\mathrm{p} K_{\mathrm{a}}$ values of 8.6 and 4.2, respectively. ADP showed poor therapeutic applications clinically because it causes irritation of the skin, dryness, a burning or stinging sensation, peeling/ exfoliation and photosensitivity with pigmentation. To overcome these limitations, various approaches have been studied such as liposomes, ${ }^{5}$ TyroSpheres, ${ }^{6}$ polymeric micelles ${ }^{7}$ and hydrogel matrices $^{8}$ to control and improve the ADP permeation. Nanovesicles have shown huge potential in topical delivery due to their advantages such as prolonged therapeutic effect and avoidance of the entry of the drug into the systemic circulation, thereby controlling the side effects. Furthermore, these nano-vesicles have been shown to provide efficacy, tolerability, compliance and cosmetic acceptability. ${ }^{1,2}$

Lipid vesicle based delivery systems have received notable attention from the scientific community due to various delivery advantages. Niosomes are non-ionic surfactant-based vesicles with a similar structure to that of phospholipid vesicle liposomes. ${ }^{9-13}$ The unique arrangement of niosomes makes it capable of encapsulating both lipophilic and hydrophilic drug molecules. In recent years, the capability of niosomes as a drug carrier has been extensively investigated. They enable the drug to be delivered at 
the target site in a controlled and/or sustained manner and have a long shelf life. ${ }^{14}$ According to their size and structure, niosomes can be administered through various routes such as intravenous, intramuscular, intraperitoneal, subcutaneous, pulmonary, ocular, oral and transdermal routes. They are also biocompatible, biodegradable and non-immunogenic in nature with low toxicity. ${ }^{15}$ Moreover, the inclusion of these vesicles in gel matrices enhances drug uptake and decreases skin irritation because of their particle size and surface composition. ${ }^{16}$

The aim of this study was to design, optimize and characterize ADP loaded niosomes. A $3^{2}$ factorial design was used to evaluate the effect of the variables such as non-ionic surfactant and lipid concentrations on mean particle size and entrapment efficiency. The drug-loaded niosomes were characterized for entrapment efficiency, particle size, and zeta potential using Fourier transform infrared spectroscopy (FTIR), differential scanning calorimetry (DSC), transmission electron microscopy (TEM), and in vitro drug release, ex vivo permeation and onemonth stability studies. Additionally, optimized niosomes were incorporated into the Carbopol gel for suitable topical applications. The niosomal gel was investigated through in vitro drug release and ex vivo permeation studies. Additionally, the niosomal gel was evaluated for in vivo skin penetration and skin irritation effects in Wistar rats and compared with the commercial formulation.

\section{Materials and methods}

\subsection{Materials}

Adapalene was gifted by Abbott Healthcare Pvt Ltd, India. Span 60 and cholesterol were purchased from Loba Chemie Pvt Ltd, India. Carbopol 934 NF was obtained from S. D. Fine Lab, Mumbai, India. The Adaferin ${ }^{\circledR}$ gel (0.1\%, Galderma India Pvt Ltd) was purchased from the local market. All other chemicals and reagents were of analytical grade.

\subsection{Animals}

Wistar male rats (National Toxicology Centre, Pune, India) weighing 180-200 $\mathrm{g}$ were utilized for the skin permeation, skin irritation and skin penetration studies. All animals were kept in specific plastic cages under standard conditions of temperature $\left(24 \pm 1{ }^{\circ} \mathrm{C}\right)$ and relative humidity (45-55\%) and $12 \mathrm{~h}$ light/dark cycles were maintained throughout the study. Rats had free access to a commercially available normal pellet diet (Pranav Agro 7 Industries, Maharashtra, India) and clean water ad libitum unless otherwise declared. The complete experiment was implemented in harmony with the guidelines of the Committee for the Purpose of Control and Supervision on Experimental Animals (CPCSEA). An experimental procedure was agreed by the Institutional Animal Ethics Committee (IAEC) of Poona College of Pharmacy, Pune (CPCSEA/26/2014).

\subsection{Preparation of adapalene loaded niosomes}

Adapalene loaded niosomes (ADP-NM) were prepared by the modified ethanol injection method. ${ }^{17}$ Briefly, different ratios of non-ionic surfactant (Span 60), cholesterol and adapalene $(10 \mathrm{mg}$ ) were accurately weighed and dissolved in $10 \mathrm{~mL}$ of acetone and ethanol $(1: 1)$ using bath sonication at $60{ }^{\circ} \mathrm{C}$. The clear organic phase was quickly injected into the aqueous phase at $60{ }^{\circ} \mathrm{C}$ under continuous stirring at $500 \mathrm{rpm}$ using a Remi magnetic stirrer utilizing a Teflon-coated bead. The aqueous phase instantly transformed into the milky phase indicating niosome formation. The whole system was subjected to evaporation at $60{ }^{\circ} \mathrm{C}$ under vacuum for $15 \mathrm{~min}$ to eliminate ethanol and the stirring was continued up to $12 \mathrm{~h}$. Water was added to maintain the volume of the niosomal suspension up to $10 \mathrm{~mL}$. The prepared niosomal dispersion was filtered through a $2-20 \mu \mathrm{m}$ filter (Ultipor GF Plus ${ }^{\circledR}$, Pall Life Sciences, CA, USA) to obtain uniform size distribution and refrigerated for $2 \mathrm{~h}$ for efficient vesicle sealing. Besides, the aqueous dispersion of ADP (ADP-AD) was formulated by dispersing accurately weighed $10 \mathrm{mg}$ of ADP in $3 \mathrm{~mL}$ of Millipore water containing $0.5 \%$ of carboxyl methyl cellulose using a magnetic stirrer (Remi Ltd, Mumbai, India) at $500 \mathrm{rpm}$ under ambient environmental conditions. ${ }^{18}$

\subsection{Effect of variables}

Based on previous understanding and preliminary data, the $3^{2}$ factorial design was utilized to optimize the amounts of Span $60\left(X_{1}\right)$ and cholesterol $\left(X_{2}\right)$, recognized as the independent variables affecting the mean particle size $\left(Y_{1}\right)$ and drug entrapment efficiency $\left(Y_{2}\right)$. The coded and actual values of the experimental design are given in Table 1 . The data obtained from various batches for mean particle size and drug entrapment efficiency were subjected to multiple regression analysis using the statistical software UNISTAT ${ }^{\mathbb{R}}$ (Statistic version 3, US).

The equation fitted was

$$
Y=\beta_{0}+\beta_{1} X_{1}-\beta_{2} X_{2}+\beta_{12} X_{1} X_{2}+\beta_{11} X_{12}-\beta_{22} X_{22}
$$

where $Y$ is the dependent variable, $\beta_{0}$ is the arithmetic mean response of the nine runs, and $\beta_{1}$ and $\beta_{2}$ are the estimated coefficients for the independent factors $X_{1}$ and $X_{2}$, respectively. The main effect terms $\left(X_{1}\right.$ and $\left.X_{2}\right)$ symbolize the average outcome of varying one factor at a time from its low to high value. The interaction terms $\left(X_{1} X_{2}\right)$ exhibit how the response alters when two factors are concurrently altered. The consequences of

Table 1 Optimization of non-ionic surfactant and cholesterol concentrations by the $3^{2}$ factorial design for the preparation of ADP-NM

\begin{tabular}{llllll}
\hline & $\begin{array}{l}\text { Coded } \\
\text { levels } \\
\left(X_{1}, X_{2}\right)\end{array}$ & $\begin{array}{l}\text { Non-ionic } \\
\text { surfactant } \\
(\mathrm{mg})\left(X_{1}\right)\end{array}$ & $\begin{array}{l}\text { Cholesterol } \\
(\mathrm{mg})\left(X_{2}\right)\end{array}$ & $\begin{array}{l}\text { Particle } \\
\text { size } \\
(\mathrm{nm})\left(Y_{1}\right)\end{array}$ & $\begin{array}{l}\text { Entrapment } \\
\text { efficiency } \\
(\%)\left(Y_{2}\right)\end{array}$ \\
\hline Batches & $-1,-1$ & 40 & 10 & $200 \pm 11.45$ & $80.54 \pm 10.99$ \\
B1 & -1 & 60 & 10 & $349 \pm 12.01$ & $88.34 \pm 11.67$ \\
B2 & $0,-1$ & 10 & $479 \pm 11.87$ & $94.23 \pm 12.01$ \\
B3 & $+1,-1$ & 80 & 20 & $259 \pm 12.54$ & $76.26 \pm 13.39$ \\
B4 & $-1,0$ & 40 & 20 & $278 \pm 13.09$ & $86.07 \pm 11.19$ \\
B5 & 0,0 & 60 & 20 & $274 \pm 12.01$ & $92.43 \pm 12.09$ \\
B6 & $+1,0$ & 80 & 20 & $218 \pm 12.23$ & $72.44 \pm 12.01$ \\
B7 & $-1,+1$ & 40 & 30 & $279 \pm 12.34$ & $84.08 \pm 13.76$ \\
B8 & $0,+1$ & 60 & 30 & $336 \pm 13.55$ & $90.23 \pm 12.55$ \\
B9 & $+1,+1$ & 80 & 30 & &
\end{tabular}

Date are mean $\pm \mathrm{SD}, n=3$. 
the variables are clarified considering the degree of coefficient and the numerical indication they carry.

\section{Characterization of adapalene loaded niosomes}

\subsection{Determination of encapsulation efficiency}

Encapsulation efficiency was estimated by isolating nonencapsulated ADP from niosomal dispersion by centrifugation at $25000 \mathrm{rpm}$ for $20 \mathrm{~min}$ at $25{ }^{\circ} \mathrm{C}$. The supernatant was suitably diluted with dimethyl sulfoxide (DMSO) and the free ADP content was calculated using a UV-vis spectrophotometer (Jasco, V-530, Japan) at $270 \mathrm{~nm}$. The entrapment efficiency (EE) was determined using the following equation: ${ }^{19}$ was visualized using a TEM coupled with DigitalMicrograph and Soft Imaging Viewer software (Olympus, Singapore).

\subsection{In vitro drug release study}

The in vitro release of ADP-NM was investigated at physiological temperature with ethanol : water $(80: 20)$ as a dissolution medium using the dialysis bag diffusion method. ${ }^{23}$ Briefly, ADP-NM equivalent to $4 \mathrm{mg}$ of ADP was placed into a dialysis bag (cellulose membrane, mol. wt. cut-off 12-140000 Da) hermetically closed and dipped into $200 \mathrm{~mL}$ of the ethanol: water $(80: 20)$ medium. The whole system was kept at $37 \pm 2{ }^{\circ} \mathrm{C}$ with constant magnetic stirring at $100 \mathrm{rpm}$. At predetermined time slots, the sample was collected and subsequently filtered through a $0.45 \mathrm{~mm}$ membrane filter, and if required suitably diluted, and the concentration of ADP was estimated spectrophotometrically at $270 \mathrm{~nm}$.

$$
\mathrm{EE}=\frac{\text { The total amount of drug }- \text { The total amount of drug in supernatant }}{\text { The total amount of drug }} \times 100
$$

\subsection{Determination of particle size and zeta potential}

The ADP-NM size was estimated using a dynamic laser diffraction system (Hydro $2000 \mathrm{SM}$, Malvern Instruments, UK) at a $90^{\circ}$ scattering angle and normal room temperature with samples aptly diluted with the dispersant water. Per sample, the mean diameter $\pm \mathrm{S}$. D. of three determinations was established using multimodal analysis. ${ }^{20}$ The surface charge was measured in triplicate with laser Doppler electrophoretic mobility measurements via a Zetasizer (NanoBrook ZetaPALS) using the 90Plus particle sizing software (ver. 394) at a temperature of $25{ }^{\circ} \mathrm{C}^{18}$

\subsection{Fourier transform infrared spectroscopy}

To study the possible chemical interactions between ADP, excipients and the formulation, Fourier transform infrared (FTIR) spectra were recorded on a Jasco V5300 FTIR (Tokyo, Japan). Samples were mixed with $\mathrm{KBr}$ to construct pellets by using a pressure of $150 \mathrm{~kg} \mathrm{~cm} \mathrm{~cm}^{-2}$. FTIR spectra were scanned in the range of $4000-400 \mathrm{~cm}^{-1}$ at a resolution of $2 \mathrm{~cm}^{-1} .^{21}$

\subsection{Differential scanning calorimetry}

A constant heating rate of $10{ }^{\circ} \mathrm{C} \mathrm{min}^{-1}$ was utilized over a temperature range of $20-340{ }^{\circ} \mathrm{C}$ for the lyophilized ADP-NM and their individual excipient components with nitrogen purging $\left(50 \mathrm{~mL} \mathrm{~min}^{-1}\right)$. Indium standards were utilized to standardize the enthalpy scale and temperature. Approximately 3-4 $\mathrm{mg}$ of samples were used for the differential scanning calorimetry (DSC) study. $^{22}$

\subsection{Transmission electron microscopy}

The surface morphology of ADP-NM was determined by utilizing transmission electron microscopy (TEM; Philips CM-200, Eindhoven, The Netherlands). ADP-NM (6-10 $\mu \mathrm{L})$ was placed over Formvar ${ }^{\circledR}$ coated copper grids (Ted Pella, Inc., Redding, $\mathrm{CA}$ ) for $5 \mathrm{~min}$, adsorbed using filter paper, and then dried at room temperature for $1 \mathrm{~h}$. The dried grid containing ADP-NM

\subsection{Ex vivo skin permeation study}

The ex vivo skin permeation of ADP from ADP-NM was studied by using the Franz diffusion cell method. ${ }^{24}$ The abdominal skin of the rat was removed, carefully excised and defatted so as to remove the subcutaneous fat. The defatted skin was cleaned several times with purified water. Skin permeation experiments were performed with vertical Franz diffusion cells with a surface area of $2.54 \mathrm{~cm}^{2}$ and a reservoir capacity of $19 \mathrm{~mL}$. The defatted skin was mounted on the Franz diffusion cell with the dermal side facing the receptor solution containing the mixture of ethanol and water $(80: 20)$ and the epidermal side in contact with the donor compartment and allowed to equilibrate for $30 \mathrm{~min}$ at $37^{\circ} \mathrm{C}$. The receptor solution was magnetically stirred at $100 \mathrm{rpm}$. The skin was treated with ADP alone and optimized ADP-NM (Batch 5) equivalent to $4 \mathrm{mg}$ ADP. The donor compartments were sealed carefully with parafilms to avoid the evaporation of the solution. At predetermined time points aliquots of the receptor medium $(1 \mathrm{~mL})$ were withdrawn, subsequently filtered through a $0.45 \mathrm{~mm}$ membrane filter and replaced with fresh acceptor medium. The collected aliquots were diluted and quantitatively analyzed using an UV spectrophotometer at $270 \mathrm{~nm}$. All measurements were performed in triplicate and their means were reported.

\subsection{Stability study}

ADP-NM were stored at room temperature $\left(25 \pm 5{ }^{\circ} \mathrm{C}\right.$ and $60 \pm$ $5 \% \mathrm{RH}$ ) and at $4{ }^{\circ} \mathrm{C}$ for 1 month. Particle size and encapsulation efficiency were investigated. Additionally, ADP-NM were visually evaluated for the presence of any precipitation. The samples were stored in USP type-1 flint vials and hermetically sealed with bromo butyl rubber plugs with aluminum caps. ${ }^{25}$

\section{Preparation of the gel}

The niosomal gel was formulated according to a previously published method. ${ }^{26}$ The gel of optimized ADP-NM (Batch 5) 
was formulated by incorporating the niosomal dispersion in a 1\% (w/w) Carbopol ${ }^{\circledR} 934$ NF gel base. Briefly, a sufficient amount of Carbopol ${ }^{\circledR} 934 \mathrm{NF}$ was gradually added to water and kept for $24 \mathrm{~h}$ for complete hydration of polymer chains. Triethanolamine $(0.1 \mathrm{~mL})$ was utilized to cause gelling as well as to neutralize the $\mathrm{pH}$ of the gel to 6-7. The optimized ADP-NM (Batch 5) and ADP alone were added slowly to the hydrated Carbopol solution to obtain the ADP-NM loaded gel (ADP-NM-G) and the ADP gel (ADP-G) with a final concentration of $0.1 \%(\mathrm{w} / \mathrm{w})$ of $\mathrm{ADP}$, respectively. Besides, in a similar manner the placebo Carbopol gel was also formulated without the addition of ADP-NM or ADP.

\section{Characterization of the gel}

\section{$5.1 \mathrm{pH}$, drug content and appearance}

The $\mathrm{pH}$ of the prepared ADP-NM-G was determined by using a digital pH meter. Drug content was determined by dissolving ADP-NM-G in DMSO followed by dilution with ethanol:water $(80: 20)$. The ADP concentration was estimated spectrophotometrically at $270 \mathrm{~nm} .^{27}$ The prepared ADP-NM-G were inspected visually for their appearance, color and homogeneity.

\subsection{Viscosity}

The viscosity of ADP-NM-G was measured using a stress control rheometer (Viscotech Rheometer, Reologica Instrument $\mathrm{AB}$, Sweden). ${ }^{27} 1 \mathrm{~g}$ of accurately weighed ADP-NM-G was placed on a viscometer plate with a diameter of $2.9 \mathrm{~cm}$ and Roto Cone with a $2.8 \mathrm{~cm}$ diameter. The viscosity was calculated through rheologic basic software (Version 5; Reologica Instrument $\mathrm{AB}$, Sweden) and the average of three readings was utilized to determine the viscosity at normal room temperature.

\subsection{Spreadability}

The spreadability study of ADP-NM-G was analyzed using wooden block and glass slide apparatus. Accurately weighed $5 \mathrm{~g}$ of ADP-NM-G was placed on the lower side of the block, the movable upper slide was placed on the top of the gel and the time was noted for the upper slide to separate completely from the assembly. ${ }^{28}$ The spreadability was determined by the formula:

$$
S=\frac{m \times l}{t}
$$

where $S$ is the spreadability, $m$ is the weight tied to the upper slide, $l$ is the length travelled by the upper slide and $t$ is the time taken to separate the slide.

\subsection{In vitro release studies}

In vitro release studies were carried out utilizing an artificial cellophane membrane (Membra-Cel ${ }^{\circledR}$ MD 34-14; cut-off: 12 kDa; Viskase Co, MS, USA). For this study, a vertical Franz diffusion cell with a reservoir volume of $32 \mathrm{~mL}$ and a surface area of $2.54 \mathrm{~cm}^{2}$ was utilized. The artificial cellophane membrane was firmly mounted between the two halves of the diffusion chamber. The receptor chamber contained ethanol: water $(80: 20)$. The whole system was kept at $37 \pm 2{ }^{\circ} \mathrm{C}$ with constant magnetic stirring at $100 \mathrm{rpm} .1 \mathrm{~g}$ of accurately weighed ADP-NM-G gel and the commercial gel were placed on the donor chamber, respectively. The donor compartments were sealed carefully with parafilms to avoid the evaporation of the solution. At predetermined time points aliquots of the acceptor medium $(1 \mathrm{~mL})$ were withdrawn, subsequently filtered through a $0.45-\mathrm{mm}$ membrane filter and replaced with fresh acceptor medium. The collected aliquots were then properly diluted and quantitatively analyzed using an UV spectrophotometer at $270 \mathrm{~nm}$. All measurements were performed in triplicate and their means were reported. The release kinetics of ADP from ADP-NM-G and the commercial gel were compared. The analysis of the data was carried out using PCPDisso software, version 3 (Poona College of Pharmacy, India).

\subsection{Ex vivo permeation study}

The ex vivo permeation study was carried out on the excised Wistar rat skin according to the study protocol approved by the IAEC constituted under the CPCSEA. The abdominal skin of the rat was removed, carefully excised and defatted so as to remove the subcutaneous fat. The defatted skin was cleaned several times with purified water. Further experimentation was similar to the method described in the 'ex vivo skin permeation study' section. Measurements were performed in triplicate and their means were reported.

\subsection{Skin irritation study}

The skin irritation potential of ADP-NM-G was investigated using the Draize patch test on rats. ${ }^{29}$ The skin irritation potential of the developed ADP-NM-G was compared with those of the commercial gel and ADP-G. In the present study, three groups of rats $(n=6)$ were used to evaluate skin irritation. The area on the back of each rat was shaved prior to the experiment. The first, second and third groups were applied with ADP-G, optimized ADP-NM-G and the commercial gel, respectively. An accurately weighed $1 \mathrm{~g}$ of each formulation was applied to the hair-free skin of rats by equally distributing within an area of $4 \mathrm{~cm}^{2}$. The skin was observed for any visible change such as erythema (redness) at 24, 48 and $72 \mathrm{~h}$ after the application of formulations. The sensitivity was scored as 0, 1, 2 and 3 for no reaction, slight patchy erythema, patchy erythema and severe erythema with or without edema, respectively. ${ }^{30}$ Additionally, the primary irritation index (PPI) was calculated using the method described by Jain et al. (2011). ${ }^{31}$

\subsection{In vivo skin permeation study}

The in vivo skin permeation of ADP was studied by using the tape-stripping method. ${ }^{32}$ The animals were divided into three groups $(n=6)$ to evaluate the skin penetration potential. In brief, the dorsal skin of the rats was shaved prior to the experiment and the first, second and third groups were applied with ADP-G, optimized ADP-NM-G and the commercial gel, respectively. A formulation equivalent to $1 \mathrm{mg}$ was applied to the hair-free skin of rats by equally distributing within an area of $2 \mathrm{~cm}^{2}$. The in vivo trials were performed in an examination room under the controlled environmental conditions of 
$25 \pm 1{ }^{\circ} \mathrm{C}$ temperature and $40 \pm 5 \% \mathrm{RH}$. At the end of the $12 \mathrm{~h}$ treatment, excess formulation was removed, and the stratum corneum (SC) surface was blotted completely dry using an absorbent pad. The site was then left untouched for $1 \mathrm{~h}$ before stripping. Then the SC was serially stripped from the same area 15 times with adhesive tape (Scotch Book Tape; 3M Tegaderm) in accordance with a previously published method. ADP in the tape strips was consequently extracted with $5 \mathrm{~mL}$ of DMSO under controlled environmental conditions. Samples were filtered through a $0.45 \mu \mathrm{m}$ membrane filter prior to the UV analysis. The sample was then appropriately diluted and quantitatively analyzed using an UV spectrophotometer at $270 \mathrm{~nm}$.

\subsection{Stability study}

The developed ADP-NM-G was packed in USP type-1 flint vials and hermetically sealed with bromo butyl rubber plugs with aluminum caps and subjected to stability studies for 6 months at room temperature $\left(25 \pm 5{ }^{\circ} \mathrm{C}\right.$ and $\left.60 \pm 5 \% \mathrm{RH}\right)$. The samples were observed periodically for any change in the physicochemical parameters including color, appearance and drug content.

\subsection{Statistical analysis}

All the experiments were performed in triplicate and displayed as mean \pm standard deviation (SD). The results obtained were subjected to Student's $t$-test and the statistical analysis was carried out using online QuickCalcs GraphPad software. Data were analyzed in the following manner: ${ }^{*} P>0.05$ (not statistically significant), ${ }^{* *} P \leq 0.05$ (statistically significant) and ${ }^{\#} P \leq 0.001$ (highly statistically significant).

\section{Results and discussion}

The high surface area of the skin (1.5-2.0 sq. m) and the advantages such as noninvasive nature, bypassing first-pass metabolism, controlled delivery of medicament, reduced dosing frequency and improved patient compliance have recognized the topical route as a better option to drugs with limited oral bioavailability. Several studies showing the potential of various novel nanocarriers in combating the barrier nature of the stratum corneum further increased the interest of formulation scientists in investigating newer ways to maximize drug delivery through the topical route. Among these various novel nanocarriers, niosomes were selected in the present investigation due to their better stability, less toxicity and cost-effectiveness with an aim to enhance the therapeutic efficacy and reduce the systemic toxicity of ADP and the results were compared with the commercial ADP topical formulation.,19,33

\subsection{Factorial design}

In the present study, niosomes have been formulated and studied as topical delivery carriers for ADP. The $3^{2}$ factorial design was performed using the Design-Expert ${ }^{\circledR}$ (Ver. 9.0, Stat-Ease, Inc., USA) program. Literature review and preliminary trials were utilized to choose the initial variables with suitable levels. ${ }^{34}$ The factorial design was used to determine the variables which have significant effects on mean particle size and (entrapment efficiency EE) using two levels with a center point in numerical variables. A classical niosome vesicle would consist of non-ionic surfactants with different HLB values that affects the EE which is also stabilized by the addition of the optimum amount of cholesterol to develop stable vesicles. A further increase of the cholesterol content beyond a certain limit decreases the EE because of the disruption of the normal bilayer arrangement, so factorial designs were used to study the most suitable ratio of the surfactant and cholesterol.

As per the $3^{2}$ factorial design, nine different batches were formulated using the modified ethanol injection method. Response surface plots constructed using the Design-Expert ${ }^{\mathbb{R}}$ program demonstrating the effect of the two significant variables as a surface in three-dimensional space are shown in Fig. 1, whereas the coded levels and actual values of the variables along with the calculated responses are revealed in Table 1 . The data obtained were subjected to multiple regression analysis using the PCPDisso software and fitted with the following equation:

$$
Y=\beta_{0}+\beta_{1} X_{1}-\beta_{2} X_{2}+\beta_{12} X_{1} X_{2}+\beta_{11} X_{12}-\beta_{22} X_{22}
$$
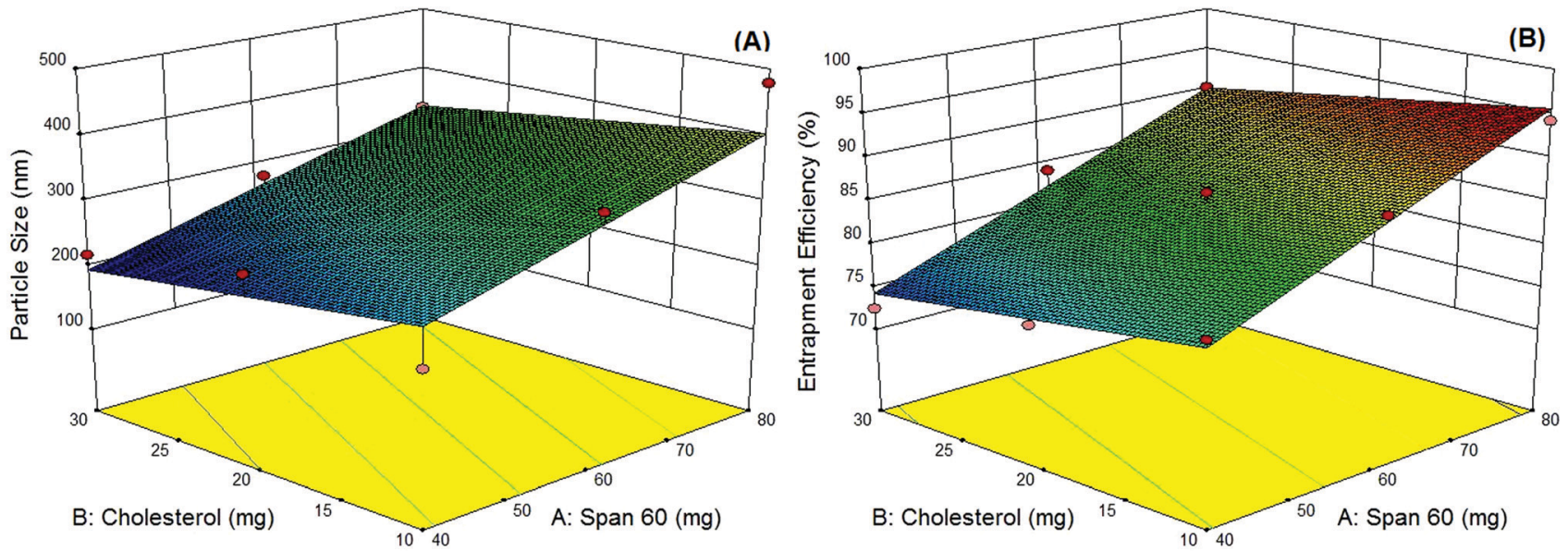

Fig. 1 Response surface plots of (a) particle size and (b) entrapment efficiency. 
where $X$ is the level of the factors, $Y$ is the measured response and $\beta$ is the coefficient computed from the responses of the formulations. The outcomes of the multiple regression analysis for particle size and EE are as follows:

$$
\begin{gathered}
Y_{\mathrm{ps}}=299.11+72.00 X_{1}-32.50 X_{2} \\
Y_{\mathrm{EE}}=+84.96+7.94 X_{1}-2.73 X_{2}
\end{gathered}
$$

Particle size is the key variable that determines the rate and extent of drug release. Moreover, it also has a significant impact on the permeation and biodistribution of a drug. The particle size, uniformity and zeta potential (Fig. 2) of the optimized ADP-NM batch were $278 \mathrm{~nm}, 0.727$ and $-17.99 \mathrm{mV}$, respectively.

The EE of the optimized ADP-NM batch was $86.07 \%$ (Table 1). It was observed that an increase in the surfactant concentration increases the EE. It may be because the high surfactant fraction controlled the diffusion rate of the drug from the concentrated exterior surfactant phase. Eventually, it offers more time for the droplet formation and thus enhances the EE. ${ }^{23}$ The mean particle size $\left(Y_{\mathrm{ps}}\right)$ and entrapment efficiency $\left(Y_{\mathrm{EE}}\right)$ were in the ranges of $218-479 \mathrm{~nm}$ and $72.44-94.23 \%$, respectively. The multiple regression analysis of the mean particle size and EE of the factorial batches revealed a good fit $\left(R^{2}=0.9796\right.$ for particle size and $R^{2}=0.9727$ for EE), suggesting the strong influences of the selected variables. After the interpretation of the developed surface plots, it was observed that Span 60 and cholesterol at the middle level (0) is better with respect to the mean particle size and EE. The optimized ADP-NM (batch 5) contains Span 60 (60 mg) and cholesterol $(20 \mathrm{mg})$. The results indicated that the observed values of the optimized formulation were very similar to the predicted values. It has been well reported that the electrostatic attraction between the carboxylic $-\mathrm{OH}$ and positively charged particles affects the charge distribution, therefore causing a reduction in the zeta potential of the whole system. This nature clearly suggests the existence of strong electric charges on the particle surfaces to obstruct the agglomeration. ${ }^{35}$ The zeta potential value indicated that the formulated ADP-NM has adequate charge and mobility to hinder the aggregation of particles. This optimized ADP-NM was preferred for further investigation.

\subsection{Fourier transform infrared spectroscopy}

The FT-IR spectra of ADP, cholesterol, Span 60 and lyophilized ADP-NM are shown in Fig. 3. The ADP showed a characteristic hydroxyl group peak at $2903 \mathrm{~cm}^{-1}$ while ADP-NM showed an absorption peak at $2918 \mathrm{~cm}^{-1}$, corresponding to the hydroxyl group peak of ADP. Furthermore, ADP also showed characteristic stretching bands within the carbonyl region $\left(1689 \mathrm{~cm}^{-1}\right)$ which was slightly shifted to the higher side $\left(1737 \mathrm{~cm}^{-1}\right)$ in ADPNM. This could be attributed to the interaction of the carbonyl group with the hydroxyl groups of cholesterol or Span 60 .

\subsection{Differential scanning calorimetry}

The differential scanning calorimetry (DSC) thermograms of ADP, cholesterol, Span 60 and lyophilized ADP-NM are shown in Fig. 4. The DSC thermograms showed that ADP exhibits a single endothermic peak at $329{ }^{\circ} \mathrm{C}$ representing its crystalline nature, while cholesterol and Span 60 melted at 150.45 and $59.20{ }^{\circ} \mathrm{C}$, respectively. The melting endotherm for ADP was not observed in the thermogram of lyophilized ADP-NM demonstrating that ADP was completely encapsulated within the cholesterol matrix of niosomes in the amorphous form.

\subsection{Transmission electron microscopy}

The TEM image of ADP-NM is shown in Fig. 5. The TEM analysis results indicate that ADP-NM are spherical in shape. The surface of niosomes is smooth and the size was found to be $<200 \mathrm{~nm}$ which is consistent with the measured particle size.

\subsection{In vitro drug release study}

The release pattern of ADP from ADP-NM depends on various experimental parameters such as particle size, the dissolution medium and the drug and polymer in the medium. The in vitro release pattern of ADP from ADP-NM is shown in Fig. 6. The release of ADP from ADP-AD was found to be more rapid and reached 99\% in $12 \mathrm{~h}$. However, the ADP release from ADP-NM exhibited a biphasic release pattern with an initial burst

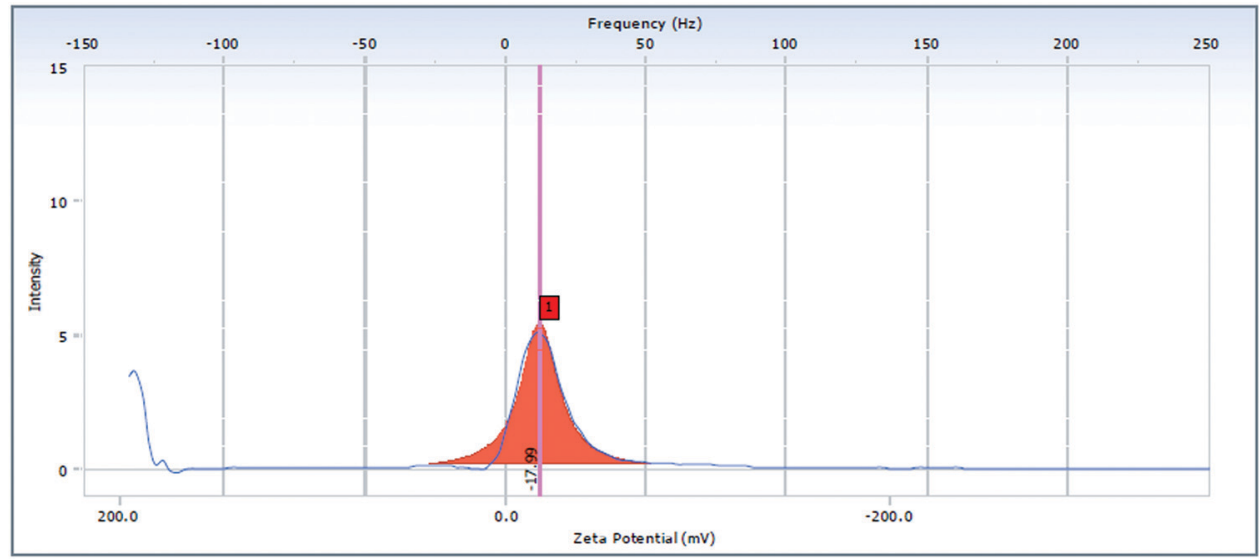

Fig. 2 Zeta potential of ADP-NM. 

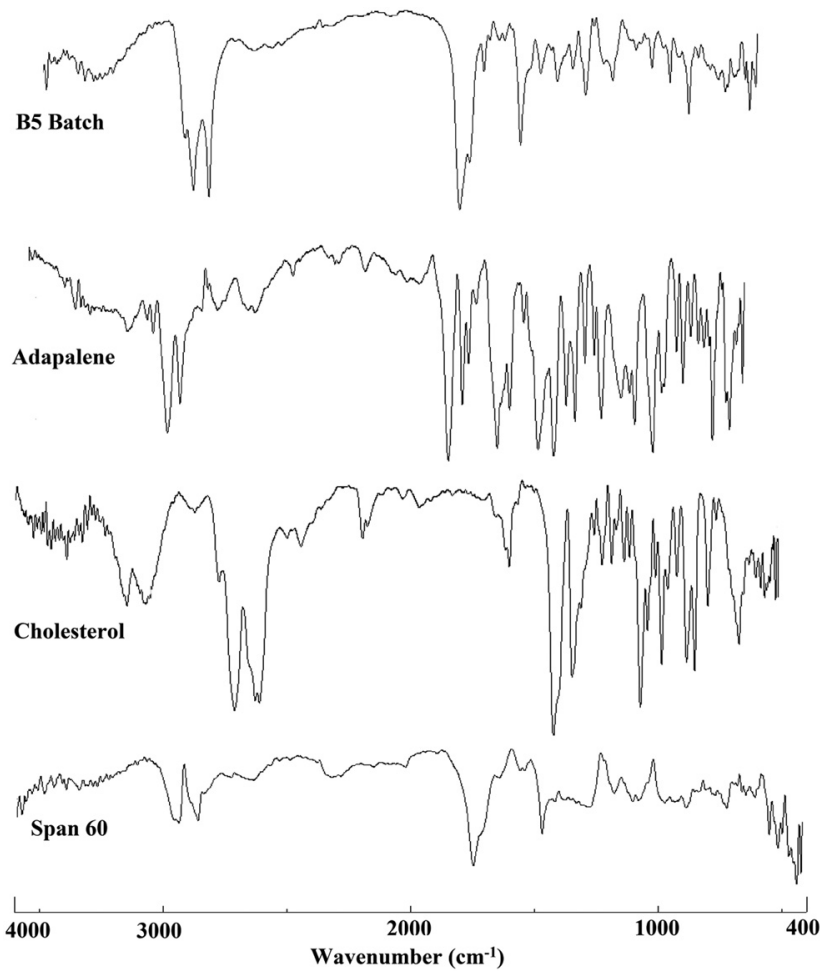

Fig. 3 FTIR spectra of Span 60, cholesterol, ADP and ADP-NM.

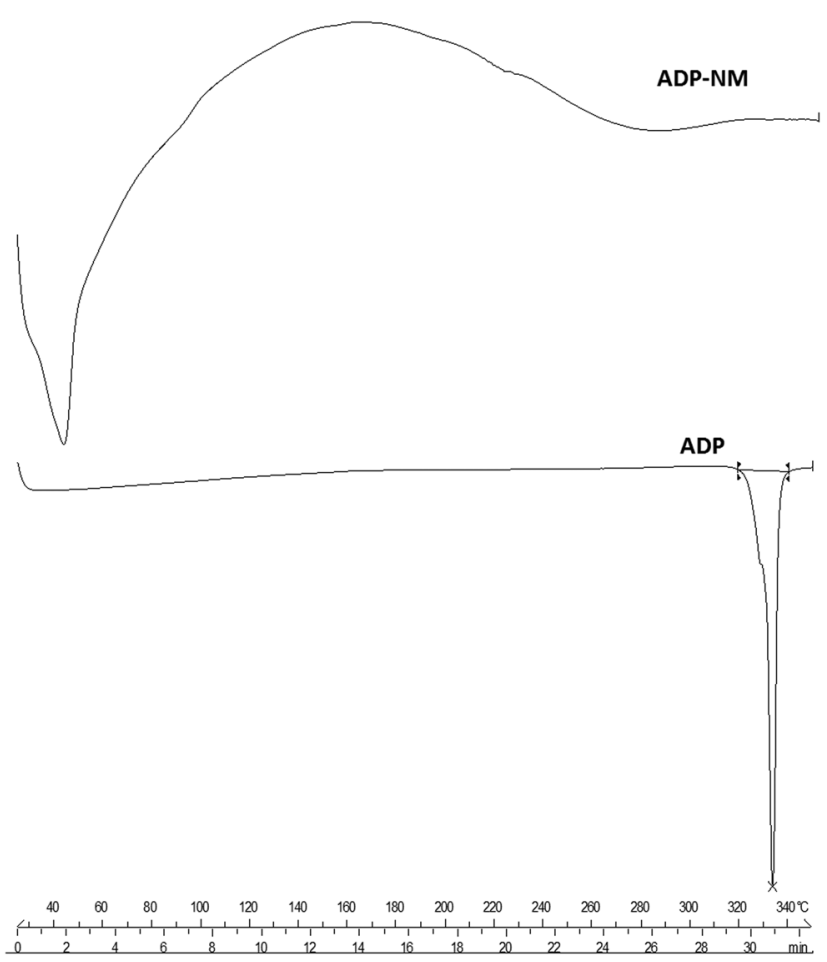

Fig. 4 DSC thermograms of ADP and ADP-NM.

release (26\%) within $1 \mathrm{~h}$, followed by a sustained release up to $12 \mathrm{~h}$ with a $73 \%$ release of ADP. This considerable difference of ADP release performance in both cases might be explained by the fact that the release of ADP from the niosomal membrane

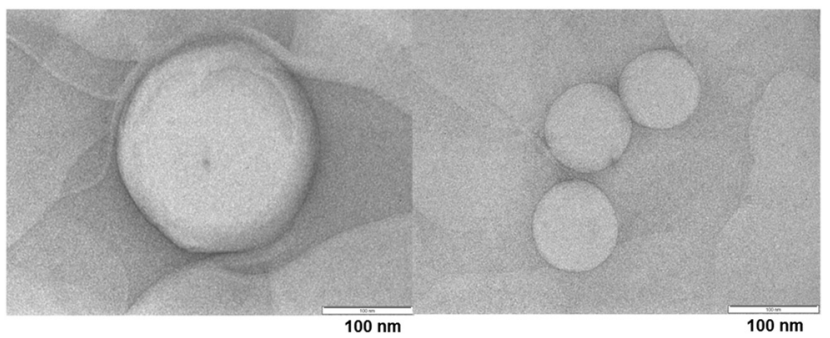

Fig. 5 Representative TEM micrographs of optimized ADP-NM (batch F5).

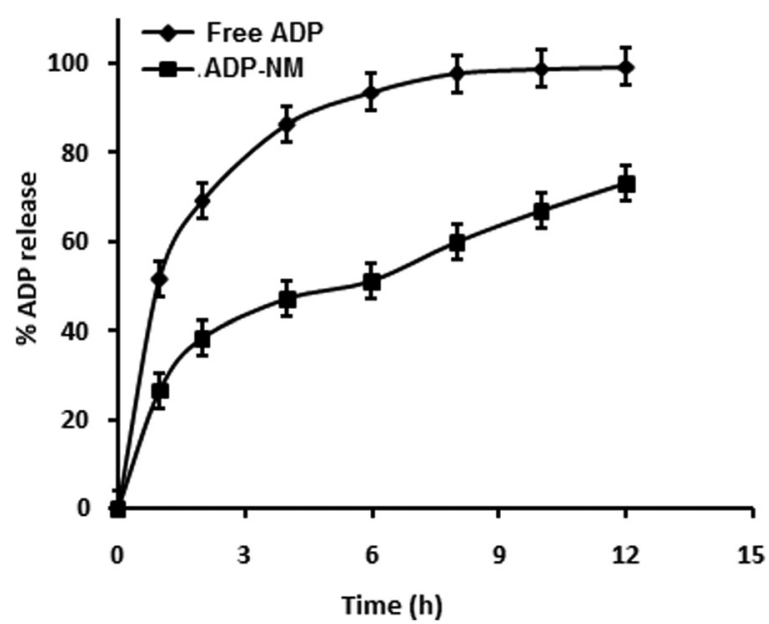

Fig. 6 Comparative in vitro release profile for free ADP and ADP-NM in ethanol: water $(80: 20)$ as a dissolution medium using the dialysis bag diffusion method. All data are means $\pm \operatorname{SDs}(n=3)$.

occurred in a more controlled manner, which can improve the penetration when applied topically.

\subsection{Ex vivo skin permeation study}

The ex vivo permeation study was carried out for ADP-AD and optimized ADP-NM (Batch 5) in order to provide better permeation profiles through the rat skin. The amount of drug permeated at the end of $24 \mathrm{~h}$ was $36.08 \%$ for ADP-AD while it was $64.31 \%$ for ADP-NM (Fig. 7). The drug permeation through the skin was slightly higher in the case of niosomes compared to ADP-AD indicating the availability of a satisfactory drug concentration at the target site which is advantageous for the effective therapy of acne.

\subsection{Stability study}

The stability study of optimized ADP-NM was carried out by subjecting the formulations to different environmental conditions and observing the changes in particle size and encapsulation efficiency as key markers of stability. The results of the stability study are shown in Table 2 . The prepared ADP-NM was found to be stable during storage over a period of 1 month. No major changes in the physicochemical parameters such as appearance and color and no precipitation were seen during storage. No statistically significant $(P>0.05)$ differences were observed in the particle size and encapsulation efficiency. 


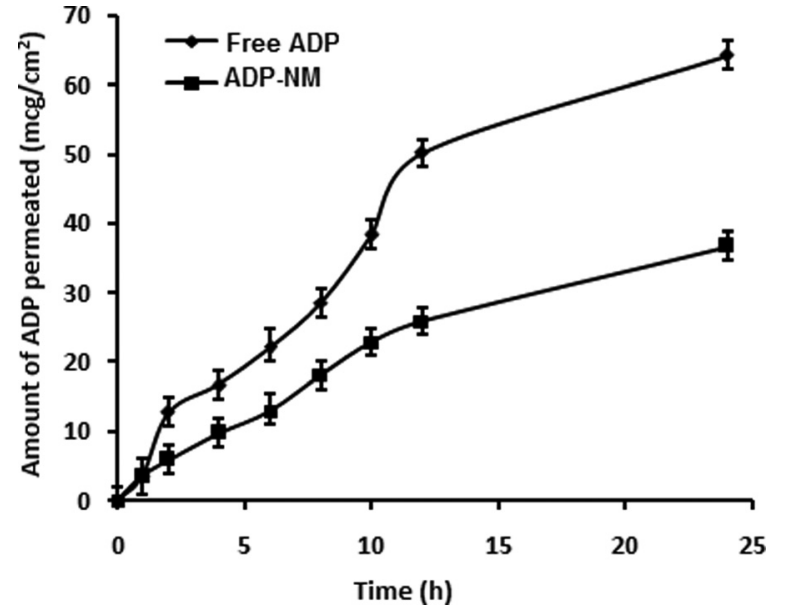

Fig. 7 Comparative ex vivo permeation study for free ADP and ADP-NM in ethanol: water $(80: 20)$ as a dissolution medium using the Franz diffusion method. All data are represented with mean $\pm \operatorname{SDs}(n=3)$.

Table 2 Stability study of optimized ADP-NM $(n=3)$

\begin{tabular}{lcc}
\hline Condition & Particle size (nm) & Entrapment efficiency (\%) \\
\hline Initial & $278 \pm 13.09$ & $86.07 \pm 11.19$ \\
$25 \pm 5{ }^{\circ} \mathrm{C}(1$ month) & $302 \pm 11.97^{a}$ & $76.26 \pm 11.08^{a}$ \\
$4 \pm 1{ }^{\circ} \mathrm{C}(1$ month) & $294 \pm 12.73^{a}$ & $79.23 \pm 10.12^{a}$ \\
${ }^{a} P>0.05$ (not statistically significant) & when compared to the initial \\
analysis.
\end{tabular}

\section{Evaluation of the gel}

\section{1 $\mathrm{pH}$, drug content and appearance}

ADP-NM-G was successfully prepared using a film hydration technique. The developed ADP-NM-G remained in the $\mathrm{pH}$ range of the skin to evade any risk of irritation upon application to the skin. The drug content was found to be $72 \pm 2.9 \%$. Furthermore, the developed ADP-NM-G was white in color, uniform and had a translucent matrix structure (Table 3).

\subsection{Viscosity and spreadability}

ADP-G and ADP-NM-G showed viscosity values of 22.01 and 23.97 $\mathrm{Pa} \mathrm{s}$, respectively. The inclusion of ADP-NM into the Carbopol $^{\circledR} 934$ NF gel base showed a satisfactory improvement in the viscosity profile as compared to ADP-G. Cholesterol particles of niosomes lead to the formation of a colloidal network that aligns itself in the direction of the applied shear, which causes an enhancement in viscosity compared to ADP-G. Moreover, the viscosity of topical gels is one of the essential physical constraints which is inversely proportional to the degree of permeation if diffusion through the vehicle is the rate-limiting step. Generally, an enhancement in the viscosity of the system would provide a more rigid structure and lessen the drug release rate and permeation. The spreadability values of ADP-G and ADP-NM-G were found to be 11.37 and $18.75 \mathrm{~g} \mathrm{~cm} \mathrm{~s}^{-1}$, respectively. ADP-NM-G showed a satisfactory improvement in spreadability as compared to ADP-G. Results show that ADPNM-G is easily spreadable by applying a small amount of shear.
Table 3 Physicochemical characteristics of ADP-G and ADP-NM-G $(n=3)$

\begin{tabular}{lllll}
\hline Batches & $\begin{array}{l}\text { Drug content } \\
(\%)\end{array}$ & $\mathrm{pH}$ value & $\begin{array}{l}\text { Viscosity } \\
(\mathrm{Pa} \mathrm{s})\end{array}$ & $\begin{array}{l}\text { Spreadability } \\
\left(\mathrm{g} \mathrm{cm} \mathrm{s}^{-1}\right)\end{array}$ \\
\hline ADP-G & $83.20 \pm 2.40$ & $6.80 \pm 1.25$ & $22.01 \pm 1.02$ & $16.45 \pm 1.34$ \\
ADP-NM-G & $76.43 \pm 2.44^{* *}$ & $7.20 \pm 2.04^{*}$ & $23.97 \pm 1.79^{*}$ & $18.75 \pm 2.11^{*}$ \\
& & &
\end{tabular}

This might be due to the unique gel matrix structure of ADPNM-G because of the presence of cholesterol vesicles.

\subsection{In vitro release studies}

The ADP release pattern from ADP-NM-G and the commercial (Adaferin ${ }^{\circledR}$ ) gel is presented in Fig. 8. ADP-NM-G showed a higher release rate (48\%) of ADP compared to the Adaferin ${ }^{\circledR}$ gel formulation (43\%), while ADP-G exhibited a higher release rate of ADP compared to ADP-NM-G (data not shown). The delayed release from ADP-NM-G was attributed to the encapsulation of ADP into niosome vesicles. The solid matrix of ADP-NM-G was responsible for drug immobilization and the subsequent lower drug release compared to ADP-G. With the fact that the encapsulation of niosomes controls the ADP release from ADP-NM-G, indicating that the integrity of niosomes is not influenced by the gel structure in which niosomes are dispersed. ${ }^{16}$ Similar release kinetics were observed for etodolac containing a topical niosomal gel system. ${ }^{28}$ This controlled release profile of ADP into the skin can modify its percutaneous absorption. Simply, they would stay on the skin surface and slowly release their content over time, resulting in better safety of the applied drugs. ${ }^{36}$

\subsection{Ex vivo permeation studies}

The ex vivo permeation study was carried out for ADP-NM-G and the commercial (Adaferin ${ }^{\mathbb{R}}$ ) gel to present better comparisons of the permeation behavior through the rat skin. The amount of

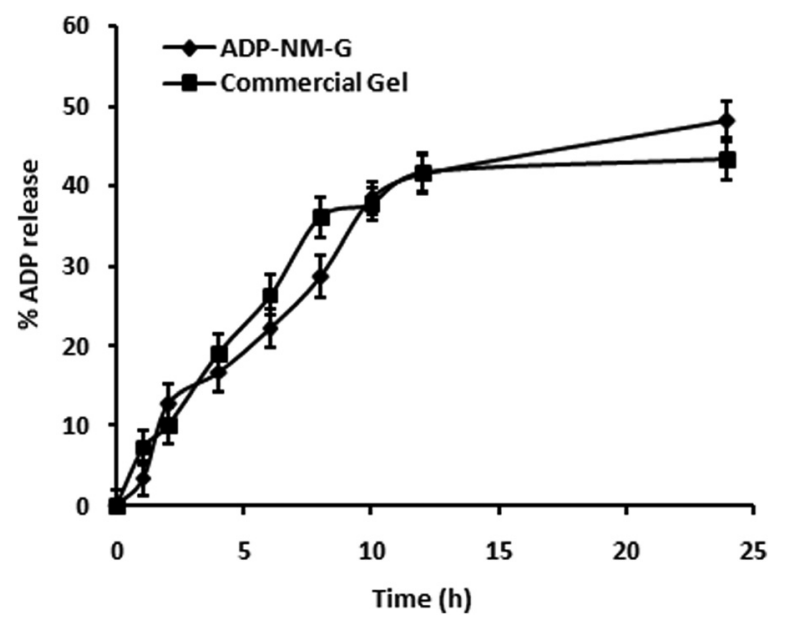

Fig. 8 Comparative in vitro release profile for ADP-NM-G and the ADP commercial gel in ethanol: water $(80: 20)$ as a dissolution medium using the vertical Franz diffusion cell method. All data are means $\pm \operatorname{SDs}(n=3)$. 


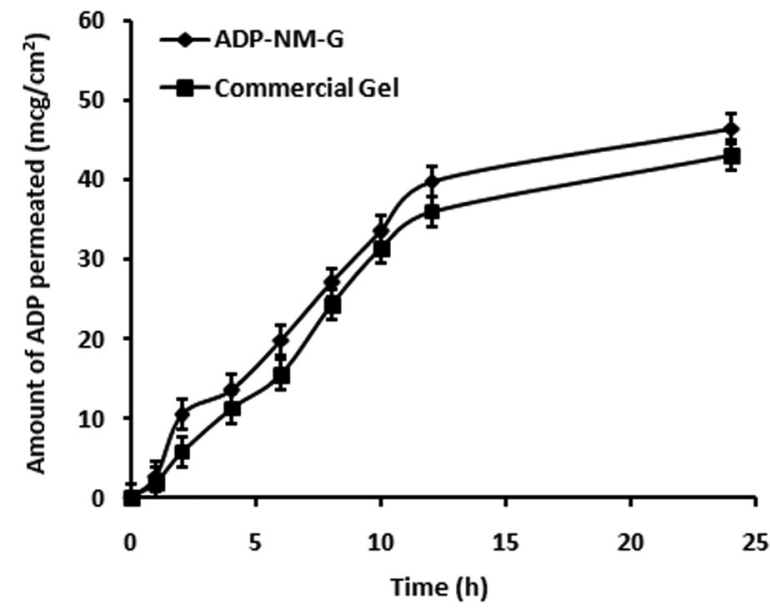

Fig. 9 Comparative ex vivo permeation study for ADP-NM-G and the ADP commercial gel in ethanol: water $(80: 20)$ as a dissolution medium using the Franz diffusion method. All data are means \pm SDs $(n=3)$.

drug permeated per unit skin surface area was plotted against time (Fig. 9). The amount of ADP permeated through the skin at the end of $24 \mathrm{~h}$ was $46.49 \%$ from ADP-NM-G whereas it was $43 \%$ from the Adaferin ${ }^{\circledR}$ gel. The drug diffusion through the skin was slightly higher in the case of ADP-NM-G as compared to the Adaferin ${ }^{\circledR}$ gel indicating the availability of a satisfactory drug concentration at the target site which is advantageous for the effective therapy of acne.

\subsection{Skin irritation study}

The outcomes of the skin irritation studies were based on the visual observation of erythema (redness). The repetitive use of
Table 4 Evaluation of primary irritation index of ADP-G, ADP-NM-G and commercial gel on Wister rats $(n=6)$

\begin{tabular}{|c|c|c|c|c|c|c|}
\hline \multirow[b]{2}{*}{ Animal no } & \multicolumn{2}{|c|}{ ADP-G } & \multicolumn{2}{|c|}{ ADP-NM-G } & \multicolumn{2}{|c|}{ Commercial gel } \\
\hline & $24 \mathrm{~h}$ & 7 day & $24 \mathrm{~h}$ & 7 day & $24 \mathrm{~h}$ & 7 day \\
\hline 1 & 1 & 1 & 0 & 0 & 1 & 1 \\
\hline 2 & 1 & 1 & 0 & 0 & 1 & 1 \\
\hline 3 & 1 & 1 & 1 & 0 & 2 & 1 \\
\hline 4 & 2 & 1 & 0 & 0 & 1 & 1 \\
\hline 5 & 1 & 1 & 1 & 0 & 2 & 1 \\
\hline 6 & 1 & 0 & 1 & 0 & 1 & 1 \\
\hline${ }^{*} \mathrm{PPI}$ & 1.16 & 0.8 & 0.5 & 0 & 1.33 & 1 \\
\hline
\end{tabular}

The sensitivity was scored as 0 : no reaction, 1 : slight patchy erythema, 2: patchy erythema and 3: severe erythema with or without edema. * PPI: primary irritation index.

ADP-NM-G exhibited negligible skin irritancy on the rat skin (Fig. 10). It is recommended that the niosomal gel formulation shows no irritation, therefore enhancing the suitability for safe topical applications. An earlier study showed the controlled release of ADP for therapeutic action, whereas the present study showed controlled release with the nonirritant nature of the niosomal gel formulation. ${ }^{37}$ Additionally, the PPI scores are shown in Table 4. ADP-G and the commercial formulation showed a PPI of around 1 while ADP-NM-G showed a PPI of less than 1. Briefly, all three groups showed PPI values less than 2 indicating that there are no signs of skin irritation. ${ }^{38}$

\subsection{In vivo skin penetration study}

The in vivo skin penetration study results are shown in Table 5. After $12 \mathrm{~h}$ the amounts of ADP recovered per area in the strips were $112,84.4$ and $46.10 \mu \mathrm{g} \mathrm{cm}^{-2}$ in the SC of rats treated with

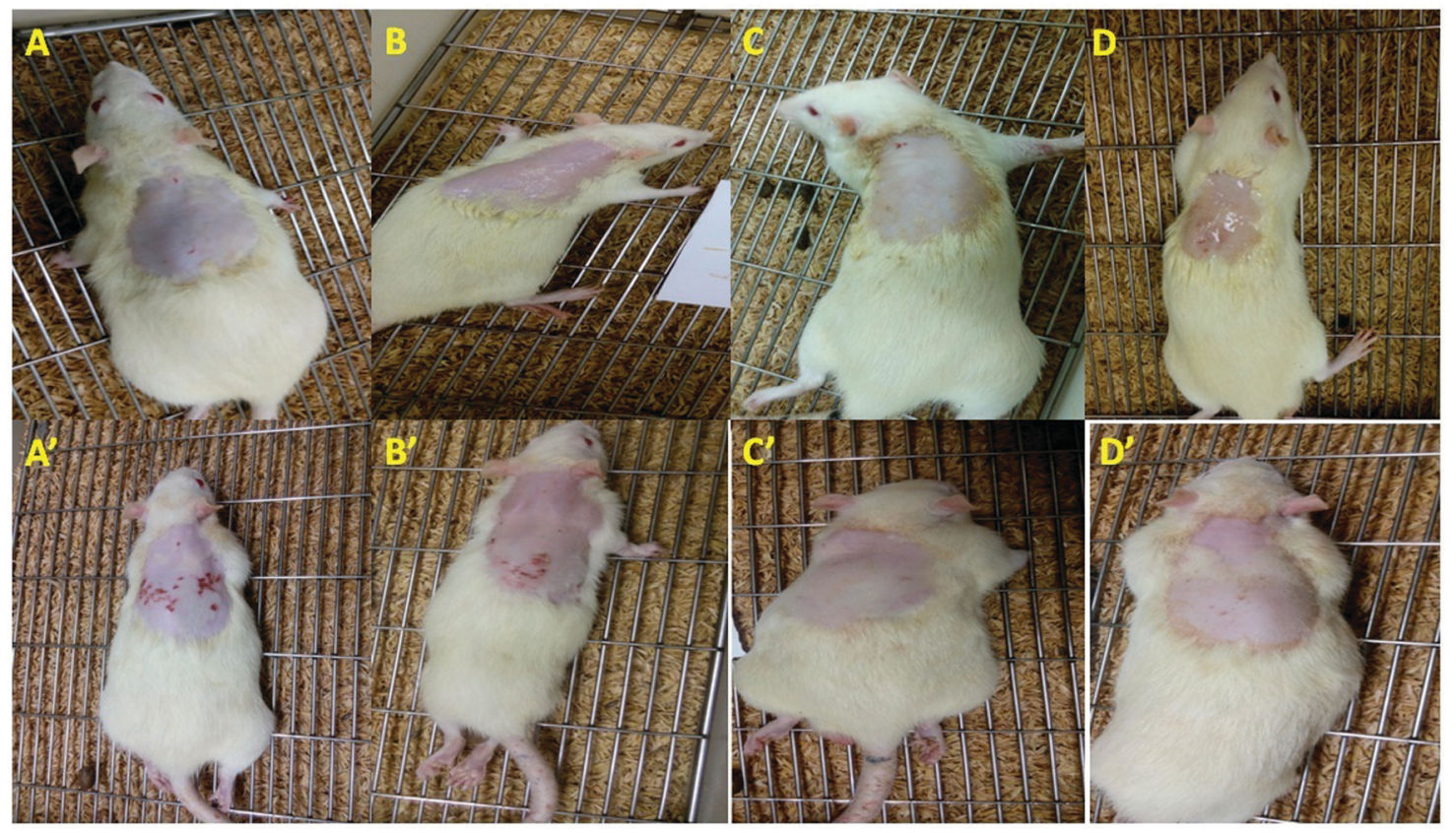

Fig. 10 Comparative skin irritation study for ADP-G, ADP-NM-G and the ADP commercial gel using the Draize patch test. After $24 \mathrm{~h}, \mathrm{~A}$ : control, $\mathrm{B}$ : ADP commercial gel, C: ADP-NM-G, and D: ADP-G; after 7 days, A': control, B': ADP commercial gel, C': ADP-NM-G, and D': ADP-G. 
Table 5 In vivo skin permeation study $(n=6)$

\begin{tabular}{ll}
\hline Formulation & Amount of ADP deposited in rat skin $\left(\mu \mathrm{g} \mathrm{cm}^{-2}\right)$ \\
\hline ADP-G & $84.49 \pm 10.22$ \\
ADP-NM-G & $112.32 \pm 11.88^{a c}$ \\
Commercial gel & $46.17 \pm 8.97^{b}$ \\
& \\
${ }^{a} P \leq 0.05$ (statistical significant) when compared to ADP-G. ${ }^{b} P \leq 0.01$ \\
(very statistical significant) when compared to ADP-G. ${ }^{c} P \leq 0.01$ (very \\
statistical significant) when compared to commercial gel.
\end{tabular}

ADP-NM-G, ADP-G and the Adaferin ${ }^{\circledR}$ gel, respectively. The ADP amounts recovered from the strips were considerably different at 1,6 and $12 \mathrm{~h}$ (data not shown). During the in vivo skin penetration study, ADP-NM-G showed significant differences of 1.32- and 2.10-fold in ADP SC residence as compared to ADP-G $(P \leq 0.05)$ and the Adaferin ${ }^{\circledR}$ gel $(P \leq 0.01)$, respectively. This indicated that the niosomal gel improved the drug residence on the skin. This outcome is in agreement with earlier investigations verifying that the use of vesicular delivery enhanced the drug residence in the SC without modifying the transdermal drug transport. ${ }^{36}$ This enhanced dermal retention of ADP was attributed to the improved contact with corneocytes and sustained release properties of niosomes. Because of their small particle size, niosomes create closer contact with the apparent junctions of corneocyte groups and channels nearby between corneocyte surfaces and leads to accumulation for several hours. ${ }^{29}$ Moreover, the high concentration of the ADP in the SC after the application of ADP-NM-G might be described by the occlusive effect, since ADP-NM-G forms a film on the skin surface that controls transepidermal water loss and favors drug penetration into the SC. ${ }^{39}$ Therefore, we can conclude that the superior ADP retention in the skin is essentially attributed to the niosomal carriers, particle size and bioadhesive characteristics of ADP-NM-G. ${ }^{29}$

\subsection{Stability study}

Similar to ADP-NM, ADP-NM-G was also subjected to stability studies. The prepared ADP-NM was found to be stable during storage over a period of 3 months. No major changes in the physicochemical parameters such as appearance and color and no precipitation were seen during storage. No notable differences were observed in the drug content (72.07\%) of ADP-NM-G after the three-month storage as compared to the initial drug content $(76.43 \%)$.

\section{Conclusion}

ADP-NM with a high EE and a low particle size were prepared effectively using a modified ethanol injection method. It was found that the concentrations of the surfactant and cholesterol play an important role in particle size and drug entrapment. Microscopic studies confirmed spherical and smooth surface niosomes in the nanometer size range. There was a sharp decrease in the crystallinity of ADP when loaded in niosomes, which was verified by DSC studies representing the complete incorporation of ADP into niosomal vesicles. ADP-NM-G demonstrated better rheological properties and control drug release up to $24 \mathrm{~h}$ as compared to the commercial gel. The higher amount of ADP deposited in the stratum corneum from ADP-NM-G shows that niosomes improved ADP residence in the skin. Additionally, ADP-NM-G showed no irritation to the skin as compared to ADP-G and the commercial formulation. Our study shows the potential of the niosomal gel for the treatment of acne. Therefore, the design and development of topical ADP-NM-G can be a novel and effective alternative to commercial formulations.

\section{Research involving human participants and/or animals}

The present work involves an animal study. The whole animal study was performed according to the guidelines of the CPCSEA (CPCSEA/26/2014). Besides, all animal study protocols were agreed by the IAEC of Poona College of Pharmacy, Pune.

\section{Conflicts of interest}

The authors do not have any conflict of interest regarding the present work.

\section{Acknowledgements}

The authors would like to thank Abbott Healthcare Pvt Ltd, India for gifting the Adapalene sample. The authors would like to acknowledge the support from Bharati Vidyapeeth Deemed to be University, Poona College of Pharmacy, Pune, Maharashtra, India.

\section{References}

1 R. Goyal, L. K. Macri, H. M. Kaplan and J. Kohn, Nanoparticles and nanofibers for topical drug delivery, J. Controlled Release, 2016, 240, 77-92.

2 H. Hamishehkar, Y. Rahimpour and M. Kouhsoltani, Niosomes as a propitious carrier for topical drug delivery, Expert Opin. Drug Delivery, 2013, 10(2), 261-272.

3 S. Piskin and E. Uzunali, A review of the use of adapalene for the treatment of acne vulgaris, Ther. Clin. Risk Manage., 2007, 3(4), 621-624.

4 L. E. Millikan, Adapalene: an update on newer comparative studies between the various retinoids, Int. J. Dermatol., 2000, 39, 784-788.

5 V. Kumar and A. Banga, Intradermal and follicular delivery of adapalene liposomes, Drug Dev. Ind. Pharm., 2016, 42(6), 871-879.

6 T. Ramezanli and B. B. Michniak-Kohn, Development and Characterization of a Topical Gel Formulation of AdapaleneTyroSpheres and Assessment of Its Clinical Efficacy, Mol. Pharming, 2018, 15(9), 3813-3822. 
7 S. G. Kandekar, S. Del Río-Sancho, M. Lapteva and Y. N. Kalia, Selective delivery of adapalene to the human hair follicle under finite dose conditions using polymeric micelle nanocarriers, Nanoscale, 2018, 10(3), 1099-1110.

8 M. A. Sallam and M. T. Marín Boscá, Mechanistic Analysis of Human Skin Distribution and Follicular Targeting of Adapalene-Loaded Biodegradable Nanospheres With an Insight Into Hydrogel Matrix Influence, In Vitro Skin Irritation, and In Vivo Tolerability, J. Pharm. Sci., 2017, 106(10), 3140-3149.

9 A. P. Pawar, S. Rajalakshmi, P. P. Mehta, K. Shaikh and C. Bothiraja, Strategies for formulation development of andrographolide, RSC Adv., 2016, 6, 69282.

10 P. P. Mehta, A. P. Pawar, K. R. Mahadik and C. Bothiraja, Emerging novel drug delivery strategies for bioactive flavonol fisetin in biomedicine, Biomed. Pharmacother., 2018, 106, 1282-1291.

11 P. P. Mehta, C. Bothiraja, S. S. Kadam and A. P. Pawar, Potential of dry powder inhalers for tuberculosis therapy: facts, fidelity and future, Artif. Cells, Nanomed., Biotechnol., 2018, 46(suppl. 3), S791-S806.

12 P. P. Mehta, C. Bothiraja, K. R. Mahadik, S. S. Kadam and A. P. Pawar, Phytoconstituent based dry powder inhalers as biomedicine for the management of pulmonary diseases, Biomed. Pharmacother., 2018, 108, 828-837.

13 P. P. Mehta, Dry Powder Inhalers: A Focus on Advancements in Novel Drug Delivery Systems, J. Drug Delivery, 2016, $1-17$.

14 D. Seleci, M. Seleci, J. Walter, F. Stahl and T. Scheper, Niosomes as Nanoparticular Drug Carriers: Fundamentals and Recent Applications, J. Nanomater., 2016, 1-13.

15 Marcos Bruschi, Strategies to Modify the Drug Release from Pharmaceutical Systems, 1st edn, Woodhead Publishing, 2015.

16 M. G. Arafa and B. M. Ayoub, DOE Optimization of Nanobased Carrier of Pregabalin as Hydrogel: New Therapeutic \& Chemometric Approaches for Controlled Drug Delivery Systems, Sci. Rep., 2017, 7, 41503.

17 K. S. Shaikh, B. Chellampillai and A. P. Pawar, Studies on nonionic surfactant bilayer vesicles of ciclopirox olamine, Drug Dev. Ind. Pharm., 2010, 36(8), 946-953.

18 P. Jadhav, C. Bothiraja and A. Pawar, Methotrexate-Loaded Nano mixed Micelles: Formulation, Characterization, Bioavailability, Safety, and In Vitro Anticancer Study, J. Pharm. Sci. Innovation, 2018, 13(3), 213-225.

19 D. Kumbhar, P. Wavikar and P. Vavia, Niosomal gel of lornoxicam for topical delivery: in vitro assessment and pharmacodynamic activity, AAPS PharmSciTech, 2013, 14(3), 1072-1082.

20 R. N. Kamble, P. P. Mehta and A. Kumar, Efavirenz SelfNano-Emulsifying Drug Delivery System: In Vitro and In Vivo Evaluation, AAPS PharmSciTech, 2016, 17(5), 1240-1247.

21 R. N. Kamble, C. Bothiraja, P. P. Mehta and V. Varghese, Synthesis, solid state characterization and antifungal activity of ketoconazole cocrystals, J. Pharm. Invest., 2018, 48(5), 541-549.
22 R. N. Kamble, R. V. Mehtre, P. P. Mehta and S. S. Patil, Albendazole Electrospun Nanofiber Films: In vitro and Ex vivo Assessment, J. Bionanosci., 2019, 9, 625-636.

23 A. P. Pawar, A. P. Gholap, A. B. Kuchekar, C. Bothiraja and A. J. Mali., Formulation and Evaluation of Optimized Oxybenzone Microsponge Gel for Topical Delivery, J. Drug Delivery, 2015, 261068.

24 T. Ramezanli, B. Kilfoyle, Z. Zhang and B. Michniak-Kohn, Polymeric nanospheres for topical delivery of vitamin D3, Int. J. Pharm., 2017, 516, 196-203.

25 Ravindra Kamble, Sumeet Sharma and Piyush Mehta, Norfloxacin mixed solvency based solid dispersions: An in vitro and in vivo investigation, J. Taibh. Univ. Sci., 2017, 11(3), 512-522.

26 A. K. Jain, A. Jain, N. K. Garg, A. Agarwal, A. Jain, S. A. Jain, R. K. Tyagi, R. K. Jain, H. Agrawal and G. P. Agrawal, Adapalene loaded solid lipid nanoparticles gel: An effective approach for acne treatment, Colloids Surf., E, 2014, 121, 222-229.

27 G. Bonacucina, S. Martelli and G. F. Palmieri, Rheological, mucoadhesive and release properties of Carbopol gels in hydrophilic cosolvents, Int. J. Pharm., 2004, 282, 115-130.

28 Gyati Shilakari Asthana, Abhay Asthana, Davinder Singh and Parveen Kumar Sharma, Etodolac Containing Topical Niosomal Gel: Formulation Development and Evaluation, J. Drug Delivery, 2016, 9324567.

29 Shilpa Shrotriya, Nisharani Ranpise, Pournima Satpute and Bhagvat Vidhate, Skin targeting of curcumin solid lipid nanoparticles-engrossed topical gel for the treatment of pigmentation and irritant contact dermatitis, Artif. Cells, Nanomed., Biotechnol., 2018, 46(7), 1471-1482.

30 N. S. Kumar Varma, P. V. Maheshwari, M. Navya, S. C. Reddy, H. G. Shivakumar and D. V. Gowda, Calcipotriol delivery into the skin as emulgel for effective permeation, Saudi Pharm. J., 2014, 22, 591-599.

31 Suresh Jain, Manish Goswami, Anil Bhandari and Vimal Arora, Skin Irritation Study of Intradermal Patch of Chitosan Containing Trazodone-HCl on Rat Skin, Int. J. Res. Pharm. Biomed. Sci., 2011, 2(3), 1082-1083.

32 J. Lademann, U. Jacobi and C. Surber, et al., The tape stripping procedure -evaluation of some critical parameters, Eur. J. Pharm. Biopharm., 2008, 72(2), 317-323.

33 K. K. Patel, P. Kumar and H. P. Thakkar, Formulation of niosomal gel for enhanced transdermal lopinavir delivery and its comparative evaluation with ethosomal gel, AAPS PharmSciTech, 2012, 13(4), 1502-1510, DOI: 10.1208/s12249012-9871-7.

34 P. P. Mehta and V. V. Dhapte, Propulsive PAT paradigm: Optimization of Freeze Drying Process, Int. J. Pharm. Sci. Rev. Res., 2014, 28(2), 240-246.

35 B. Nasseri, Effect of cholesterol and temperature on the elastic properties of niosomal membranes, Int. J. Pharm., 2005, 300(1-2), 95-101.

36 C. Bothiraja, A. D. Gholap, K. S. Shaikh and A. P. Pawar, Investigation of ethyl cellulose microsponge gel for topical 
delivery of eberconazole nitrate for fungal therapy, Ther. Delivery, 2014, 5(7), 781-794.

37 R. Najafi-Taher, B. Ghaemi and A. Amani, Delivery of adapalene using a novel topical gel based on tea tree oil nano-emulsion: Permeation, antibacterial and safety assessments, Eur. J. Pharm. Sci., 2018, 120, 142-151.

38 V. Naga Sravan Kumar Varma, P. V. Maheshwari, M. Navya, S. C. Reddy, H. G. Shivakumar and D. V. Gowda, Calcipotriol delivery into the skin as emulgel for effective permeation, Saudi Pharm. J., 2014, 22(6), 591-599.

39 C. Mota Ade, Z. M. de Freitas, E. Ricci Júnior, G. M. Dellamora-Ortiz, R. Santos-Oliveira, R. A. Ozzetti, A. L. Vergnanini, V. L. Ribeiro, R. S. Silva and E. P. dos Santos, In vivo and in vitro evaluation of octyl methoxy-cinnamate liposomes, Int. J. Nanomed., 2013, 8, 4689-4701. 